62) $\mathrm{mm}$, and LV end-systolic dimensions (LVESD) of 46.1 (30-55) $\mathrm{mm}$.

Regarding management, all patients received pulse steroids, 7 were treated with IV cyclophosphamide, 3 started mycophenolate mofetil, and 1 patient underwent 3 sessions of immunoadsorption.

Follow-up echocardiography after 3 months showed a mean LVEF of $42.8(15-66) \%$, LVEDD of $52(42-62) \mathrm{mm}$, and LVESD of $38.9(22-55) \mathrm{mm}$.

Regarding outcomes, 4 patients had a complete recovery and normalization of LVEF, all of them were females, 3 presented with cardiogenic shock and 1 with acute heart failure, with prompt initiation of immunosuppressive therapy. 2 patients became asymptomatic but achieved partial LVEF improvement. Mean duration to LVEF improvement in all patients was $2.7(1-4)$ weeks. 4 patients failed to respond to treatment, three of them died within 3-months of LM diagnosis, including the 2 males and those on MHD, all 3 had biopsy-proven class IV LN.

Conclusion It is crucial to recognize LM in the setting of acute heart failure \& cardiogenic shock in SLE patients, as early diagnosis and prompt treatment with pulse steroids and other immunosuppressive drugs may achieve complete remission of myocarditis. Male gender with class IV LN biopsy proven or on MHD may be useful as predictors of poor prognosis.

\section{P116 OUTCOMES AND SAFETY OF RITUXIMAB USE IN SYSTEMIC LUPUS ERYTHEMATOSUS - A SINGLE-CENTRE ANALYSIS}

Daniel G Oliveira, Raquel Faria, Flávio Pereira, Luciana Faria, Ana Campar, Mariana Brandão, Isabel Almeida, António Marinho, Fátima Farinha, Carlos Vasconcelos. Dept. of Internal Medicine, Centro Hospitalar Universitário do Porto (CHUP), Unidade de Imunologia Clínica, CHUP, UMIB - ICBAS-UP, Unidade de Imunologia Clínica Resident, CHUP, Portugal

\subsection{6/lupus-2020-eurolupus. 160}

Background/Purpose Rituximab (RTX) has been used worldwide in moderate to severe Systemic Lupus Erythematosus (SLE), despite failure in clinical trials. We reviewed our centre's experience in efficacy, tolerability and safety of RTX in SLE patients.

Methods Retrospective single-centre (35 year long, 700 SLE patient cohort) review of records of all SLE adults treated with RTX from 2009 until September 2019. Outcomes were based on physician's assessment, SLEDAI variation, drug reactions, infections, neutrophil count, immunoglobulin and B-cell count.

Results 45 patients $(6,4 \%$ of total) were treated with RTX, 38 had sufficient data for analysis. Thirty (93,8\%) female, mean diagnosis age 30,5 years, mean disease duration at first RTX of 123,1 months ( \pm 119 ). Five patients had more than one induction and 11 patients had maintenance doses - total 63 administrations of RTX. Induction regimen was mainly 1 g 15 days apart. The main indications for treatment were lupus nephritis $(n=12)$, arthritis $(n=7)$ and skin involvement $(n=6)$. Mean pre-treatment SLEDAI was 9,86 $\pm 6,4$ points. Most patients had a favorable response $(84,2 \%, \mathrm{n}=32)$ with a mean SLEDAI reduction of 7,2 points $( \pm 5,2)$. B-cell depletion at 3 or 6 months
$(52,9 \%)$ was more frequent in responders $(p=0,003)$, but 8 non-depleters also responded. Non-responders had lower C3 and hemoglobin pre-RTX $(\mathrm{p}<0,05)$. Hypersensitivity reactions occurred 3 times (during the first cycle), 1 requiring adrenaline. One patient had a late-onset allergic reaction. Other adverse outcomes included infection requiring hospitalization $(7,9 \%, \mathrm{n}=5)$, non-serious infection $(6,4 \%, n=4)$, non-severe neutropenia $(3,2 \%, n=2)$, acute heart failure $(1,6 \%, \mathrm{n}=1)$ and death due to serious infection $(1,6 \%, n=1)$. There were 2 cases $(3,2 \%)$ of $\operatorname{IgG}$ hypogammaglobulinemia.

Conclusion Our centre has a higher use of RTX than that reported in the literature. Success rate for RTX is high in our cohort with very few serious adverse events.

\section{\begin{tabular}{|l|l}
\hline P117 RITUXIMAB THERAPY FOR PRIMARY SJÖGREN'S \\
\hline
\end{tabular} SYNDROME - A RETROSPECTIVE SINGLE-CENTRE STUDY}

${ }^{1}$ Mariana Figueiras, ${ }^{1}$ Filipa Sousa, ${ }^{2,3}$ Mariana Brandão, ${ }^{4}$ Daniel Oliveira, ${ }^{2,3}$ Raquel Faria, ${ }^{2}$ Ana Campar, ${ }^{2,3}$ Isabel Almeida, ${ }^{2,3}$ António Marinho, ${ }^{2}$ Fátima Farinha, ${ }^{2,3}$ Carlos Vasconcelos. 'Unidade de Imunologia Clínica resident, Centro Hospitalar Universitário do Porto, Porto; '2Unidade de Imunologia Clínica, Centro Hospitalar Universitário do Porto, Porto; ${ }^{3}$ UMIB-ICBAS-Universidade do Porto, Porto; ${ }^{4}$ Internal Medicine, Centro Hospitalar Universitário do Porto, Porto, Portugal

\subsection{6/lupus-2020-eurolupus. 161}

Background The rationale for B cell depletion therapy with rituximab (RTX) in primary Sjögren's syndrome (pSS) relies upon the well-established role of B cell hyperactivity in immunopathogenesis. We reviewed our centre's experience in efficacy, tolerability and safety of RTX in pSS patients.

Methods Retrospective single-centre (35 years long, 115 pSS patients cohort) observational study of RTX use in pSS adults from 2006 until September 2019, based on medical records, with data concerning indication and duration of treatment. Outcomes were assessed by subjective physician's perspective, ESSDAI variation, drug reactions, infections, neutrophil count, immunoglobulin and B-cell count. ESSDAI scores were calculated for pre and post whenever possible.

Results Ten female pSS patients were treated with RTX, with an average diagnosis age of 50,7 years and an average follow-up time of 5,6 years. Indications for RTX were: 3 peripheral nervous system (NS) manifestations, 3 central NS manifestations, 1 vasculitis, 1 vasculitis, central NS and macrophagic activation syndrome, 1 disabling musculoskeletal manifestations and 1 interstitial lung disease. Six patients became asymptomatic (4 of them with CD19 depletion), 2 did not experienced any benefit (1 with CD19 depletion) and 2 had symptomatic improvement (1 with CD19 depletion). Two patients had severe adverse reactions to rituximab (anaphylactic reaction and sweet syndrome). Although they needed hospital admission, they were able to recover completely. Three patients developed serum sickness. There were no cases of hypogammaglobulinemia or neutropenia after the treatment.

Conclusions Despite of the scarcity of studies validating its use, RTX can be considered for severe or refractory pSS involvement, with a reasonable safety profile. 